\title{
Introduction to the Special Section of the Asia-Pacific Symposium on Measurement of Mass, Force and Torque (APMF) 2015
}

\author{
Min-Seok Kim \\ Korea Research Institute of Standrads and Science, 267 Gajeong-Ro, Yuseong-Gu, Daejeon 34113, Republic of Korea
}

\begin{abstract}
Section: EDITORIAL
Citation: Min-Seok Kim, Introduction to the Special Section of the Asia-Pacific Symposium on Measurement of Mass, Force and Torque (APMF) 2015, Acta IMEKO, vol. 6, no. 2, article 1, July 2017, identifier: IMEKO-ACTA-06 (2017)-02-01
\end{abstract}

Section Editor: Paul Regtien, Measurement Science Consultancy, The Netherlands

Received June 15, 2017; In final form June 15, 2017; Published July 2017

Copyright: (C) 2017 IMEKO. This is an open-access article distributed under the terms of the Creative Commons Attribution 3.0 License, which permits unrestricted use, distribution, and reproduction in any medium, provided the original author and source are credited

Corresponding author: Min-Seok Kim, e-mail: minsk@kriss.re.kr

\section{Dear Readers,}

the Asia-Pacific Symposium on Measurement of Mass, Force and Torque (abbreviated as APMF) is a biannual symposium held in the Asia-Pacific region (mostly in China, Japan and Korea). The APMF was firstly held in 1992 just as a small joint symposium between China and Japan (at that time, it was called as International Symposium on Measurement of Force and Mass, abbreviated as ISMFM) to exchange technical information and expertise in the area of mass, force and torque measurement. Over the last two decades, however, it has developed into a most active symposium in the Asia-Pacific region, where almost 100 experts get together to share up-todate information and discuss current issues in mass, force and torque measurement. IMEKO Technical Committee number 3 (TC-3) on "Measurement of Force, Mass and Torque" has sponsored the APMF symposium with a partnership since the first joint conference between IMEKO TC-3 and APMF was held in 1998 in Daejeon, Korea.

The recent APMF symposium was held in Seoul, Korea in October 2015. It was a successful symposium contributed by almost 90 participants from 10 countries presenting 35 scientific papers. In this Special Issue, you can find 12 articles selected by the International Program Committee of the APMF, which is considered to be worth of publishing in Acta IMEKO journal after peer reviewing.

The papers included in the special issue show recent progress of the research on the measurements in the field of mass, force and torque in the Asia-Pacific region. Among 12 articles, the number of papers whose topic belongs to mass, force and torque is 5, 3 and 4, respectively. More in detail, in the topic of mass metrology, two papers from NIST, authored by Eric and Corey for each, present recent results on the development of a mass calibration system using a magnetic suspension balance for vacuum-to-air dissemination of mass scale in response to the upcoming change in the definition of the kilogram. The volume of mass artefacts must be known accurately for determination of their density. Manhong $\mathrm{Hu}$ presents a NIM volume measurement system of mass standards using an acoustic method. Yuji Yamakawa introduced a modelling of the floor vibration effect, which could contribute to the improvement of a high-speed mass measurement system, usually used to control processes in the factories. Interesting and valuable information about the long history of mass comparators over 19 years is presented by Masasaki Ueki. This work results from his dedicated long-year service in the field of mass metrology at NMIJ/AIST, Japan.

In the field of force metrology, you can find recent activities and results on small force metrology of NIM, China (Hu Gang and Jile Jiang). NIM started small force research lately compared to other National Metrology Institutes (NIST, PTB, KRISS, NPL and CMS/ITRI), but it seems that NIM is catching up rapidly the former researches. Hu Gang reports the inter-laboratory comparison results among NIM, PTB and KRISS, which show the degree of equivalence in determination of spring constants of micro-cantilevers used in atomic force microscopy while Jile Jiang introduces the first results of the NIM's electrostatic force system. On the other hand, a practical approach for estimating pressure distribution during the metal press casting process is introduced by Ryosuke Tasaki through 
a measurement of molten metal's reaction force. It is very difficult to measure pressure directly inside casting molds due to their temperature, so the proposed technique could provide a practical solution to know the pressure distribution inside the casting molds.

Torque metrology is a fast-growing field in Asia. Over the last 10 years, several NMIs in Asia newly established torque standards in response to increasing demands from industries. This issue includes 4 articles in this topic. Zhimin Zang introduces a recently developed small torque standard machine with $1 \mathrm{~N} \cdot \mathrm{m}$-capacity. NMIJ is one of key players of torque metrology in Asia. Koji Ogushi reports inter-comparison results of torque standards between NMIJ and KRISS in the range from $50 \mathrm{~N} \cdot \mathrm{m}$ to $2000 \mathrm{~N} \cdot \mathrm{m}$. NMIJ and KRISS firstly conducted a bilateral comparison in 2004. After the first comparison, the bilateral comparison was conducted again in 2010 to confirm the improvement of uncertainty of the $1 \mathrm{kN} \cdot \mathrm{m}$ deadweight NMIJ torque machine. The $2 \mathrm{kN} \cdot \mathrm{m}$ torque range was selected to check the stability of the $20 \mathrm{kN} \cdot \mathrm{m}$ deadweight torque machine in NMIJ. Atsuhiro Nishino presented the results of intra-laboratory comparisons of reference torque wrenches in Japan. Reference torque wrenches are popularly used for calibration of torque wrench testers, calibrators, and torque wrenches. This paper can give you information about the calibration capabilities of reference torque wrenches. Finally, in a paper presented by Tassanai Sanponpute, NIMT introduces torque transducers with an additional (the second) strain gauge bridge attached on the second spring body whose stiffness is at least 2 times higher than that of the first spring body where the first bridge is attached to produce a measurement signal. The second bridge produces a check signal to be used for monitoring the stability of torque transducers. Through a series of overloading tests, he demonstrated the function ratio defined by a ratio of outputs of the measurement signal and the check signal could be a useful factor to check the stability.

I would like to give my sincere thanks to all contributors, Editors, authors and reviewers, who make this issue possible and hope that you will enjoy reading this Special Section. 